\title{
Optimal Channel Assignments for Lattices with Conditions at Distance Two *
}

\author{
Jerrold R. Griggs and Xiaohua Teresa Jin \\ Mathematics Department, University of South Carolina, Columbia, SC 29208, USA \\ griggs@math.sc.edu,jin2@math.sc.edu
}

\begin{abstract}
The problem of radio channel assignments with multiple levels of interference can be modeled using graph theory. Given a graph $G$, possibly infinite, and real numbers $k_{1}, k_{2}, \ldots, k_{p} \geq 0, a$ $L\left(k_{1}, k_{2}, \ldots, k_{p}\right)$-labeling of $G$ assigns real numbers $f(x) \geq 0$ to the vertices $x$, such that the labels of vertices $u$ and $v$ differ by at least $k_{i}$ if $u$ and $v$ are at distance $i$ apart. We denote by $\lambda\left(G ; k_{1}, k_{2}, \cdots, k_{p}\right)$ the infimum span over such labelings $f$. We survey this new theory of real number labelings. When $p=2$ it is enough to determine $\lambda(G ; k, 1)$ for reals $k \geq 0$, which will be a piecewise linear function. We present the function for the square lattice (grid) and for the hexagonal lattice. For the triangular lattice, we have also solved it except for the range $1 / 2 \leq k \leq 4 / 5$.
\end{abstract}

\section{Introduction}

As wireless networks continue to grow rapidly and the radio frequency spectrum remains a scarce resource, efficient channel assignment algorithms are increasingly important.

The channel assignment problem is to assign channels to the transmitters in a network in a way which avoids interference and uses the spectrum as efficiently as possible. We consider the version suggested by Roberts (see [12]) in which the assignment must satisfy some separation constraints depending on the distance, and the goal is to make the assignment bandwidth as small as possible. The problem is modeled nicely with graph theory by letting each transmitter correspond to a vertex and representing by an edge each pair of nearby transmitters.

A $L\left(k_{1}, k_{2}, \cdots, k_{p}\right)$-labeling of a graph $G$ is an assignment of nonnegative numbers to the vertices of $G$, with $x \in V(G)$ labeled by $f(x)$, such that $|f(u)-f(v)| \geq k_{i}$ if $u$ and $v$ are at distance $i$ apart.

\footnotetext{
*Research supported in part by NSF Grant DMS-0302307
}

We denote by $\lambda\left(G ; k_{1}, k_{2}, \cdots, k_{p}\right)$ the infimum span over such $f$, i.e., the $L\left(k_{1}, k_{2}, \cdots, k_{p}\right)$-labeling number of graph $G$, where the span is the difference between the supremum and the infimum of the labels $f(x)$.

Griggs and Yeh [12] (1992) introduced integer $L\left(k_{1}, k_{2}, \ldots, k_{p}\right)$-labelings of graphs, where all labels are integers, and they obtained many results for the particular case $L(2,1)$.

The frequency channel separations $k_{i}$ for two transmitters are often inversely proportional to the distance $i$ between them, so that many articles assume $k_{1} \geq k_{2} \geq \ldots \geq k_{p}$. But this is not required in our theory. Here we consider $L\left(k_{1}, k_{2}, \ldots, k_{p}\right)$ for all nonnegative real numbers $k_{1}, k_{2}, \ldots, k_{p}$.

Wireless networks include cellular mobile networks, wireless computer networks [3], wireless ATM networks [17], and private mobile radio networks [23]. Bertossi, Maurizio and Bonuccelli [3](1995) introduced an integer "control code" assignment in Packet Radio Networks to avoid hidden terminal interference. This occurs for stations (transmitters), which that are outside the hearing range of each other, that transmit to the same receiving stations (transmitters): It is the $L(0,1)$ graph-labeling problem. Another engineering problem is to assign time slots without interference [2], which can be modeled very well by graph labelings. Different channel assignment problems in the frequency, time and code domains (with a channel defined as a frequency, a time slot [2], or a control code [3], resp.) can be modeled by graph labelings. Ramanathan [20] mentions a unified framework of channel assignments motivated by the similarity of the constraints across these domains.

This article concentrates on the minimum spans for $\Delta$-regular planar lattices (grids), with $\Delta=3,4$ or 6 , with conditions at distance two $(p=2)$. 


\section{Real Number Graph Labelings}

Since we can use any frequencies (channels) in the available continuous frequency spectrum, not only from a discrete set, we extend the idea of integer graph labelings to allow the labels and constraints $k_{i}$ to be nonnegative real numbers.

To describe optimal real number labelings of graphs, we define the $D$-set for $k_{1}, k_{2}, \ldots, k_{p}$ to be the set of linear combinations $\sum_{i} a_{i} k_{i}$ with nonnegative integer coefficients $a_{i}$. We prove the existence of some optimal labeling $f \in L\left(k_{1}, k_{2}, \ldots, k_{p}\right)(G)$ with smallest label 0 and all labels in the $D$-set, and hence the span $\lambda\left(G ; k_{1}, k_{2}, \ldots, k_{p}\right)$ exists in the $D$ set, with minimum instead of infimum, for $G$ being any graph with finite maximum degree. We cannot ensure the existence of $\lambda\left(G ; k_{1}, k_{2}, \ldots, k_{p}\right)$ for an infinite graph $G$ without some restriction, such as on the degrees.

Theorem 1 (The $D$-set Theorem). Let $G$ be a graph, possibly infinite, with maximum degree $\Delta$. Let $k_{i} \in[0, \infty), i=1,2, \ldots, p$. Then there exists an optimal $L\left(k_{1}, k_{2}, \ldots, k_{p}\right)$-labeling

$f^{*}: V(G) \rightarrow[0, \infty)$ in which the smallest label is 0 and all labels are in the $D$-set for $k_{1}, k_{2}, \ldots, k_{p}$. Hence, $\lambda\left(G ; k_{1}, k_{2}, \ldots, k_{p}\right) \in D_{k_{1}, k_{2}, \ldots, k_{p}}$.

Due to Theorem 1, all previous optimal integer labeling results are compatible with our optimal real number labeling results.

In [16] we prove $\lambda\left(G ; k_{1}, k_{2}, \ldots, k_{p}\right)$ is a nondecreasing, continuous function of real numbers $k_{i}$ for a graph $G$ with finite maximum degree. We also prove that $\lambda\left(G ; k_{1}, k_{2}, \ldots, k_{p}\right)$ is a piecewise linear function of $k_{i}$ with nonnegative integer coefficients and finitely many linear pieces for arbitrary $p$ and any finite graph $G$, or for any infinite graph $G$ with finite maximum degree when $p=2$.

Our definition gives us the following useful concept.

The Scaling Property. We have $\lambda\left(G ; d \cdot k_{1}, d \cdot k_{2}, \ldots, d \cdot k_{p}\right)=d \cdot \lambda\left(G ; k_{1}, k_{2}, \ldots, k_{p}\right)$ for real numbers $d, k_{i} \geq 0, i=1,2, \ldots, p$.

In particular, for $k_{2}>0, \lambda\left(G ; k_{1}, k_{2}\right)=$ $k_{2} \lambda(G ; k, 1)$, where $k=k_{1} / k_{2}$. We will give the minimum label span $\lambda(G ; k, 1), k \geq 0$ for some infinite regular lattices.

\section{The Triangular Lattice}

In a radio mobile network, large service areas are often covered by a network of nearly congruent polygonal cells, with each transmitter at the center

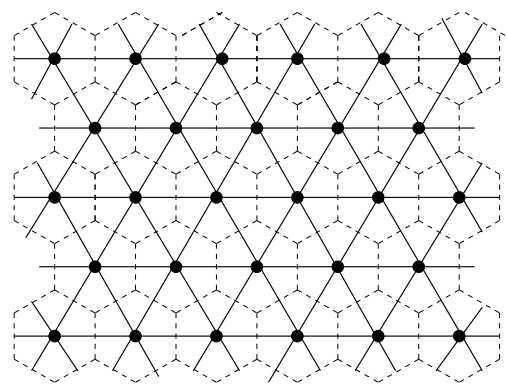

Figure 2: The Hexagonal Cell Covering

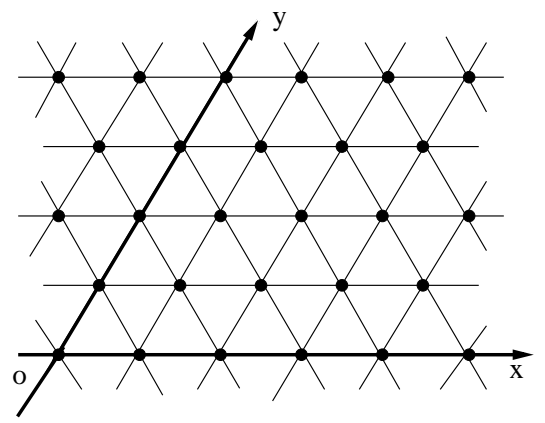

Figure 3: The Triangular Lattice $\Gamma_{\triangle}$

of a cell that it covers. A honeycomb of hexagonal cells provides the most economic covering of the whole plane [9] (i.e., a cover of the whole plane with smallest possible transmitter density), where the transmitters are placed in the triangular lattice $\Gamma_{\triangle}$ (see Figure 2). We fix a point to be the original point $o$ and impose a xoy coordinate system so that one can name each point by its xoy coordinate.

Griggs [13] formulated an integer $L(k, 1)$ labeling problem on the triangular lattice $\Gamma_{\triangle}$ for the 2000 International Math Contest in Modeling. Among 243 teams which worked on this problem in four days, five teams $[5,8,11,19,21]$ won the contest and got their papers published. All winners found $\lambda\left(\Gamma_{\triangle} ; k, 1\right)$ for $k=2,3$, and some gave optimal labelings for $k=1$ or for integers $k \geq 4$ (without proving the lower bound). Goodwin, Johnston and Marcus [11] gave the exact results for integers $k \geq 4$, as well as for $\lambda\left(\Gamma_{\triangle} ; k_{1}, k_{2}\right)$ for integers $k_{1}>6 k_{2}$. Subsequently, Yeh [15] and Zhu and Shi [24] solved some more special cases for integers $k_{1} \geq k_{2}$. Calamoneri [7] stated the minimum integer span for the triangular lattice for integers $k_{1} \geq 3 k_{2}$ and gave bounds for $k_{2} \leq k_{1} \leq 3 k_{2}$, independently of us.

Here we describe the full solution of the $L(k, 1)$ - 


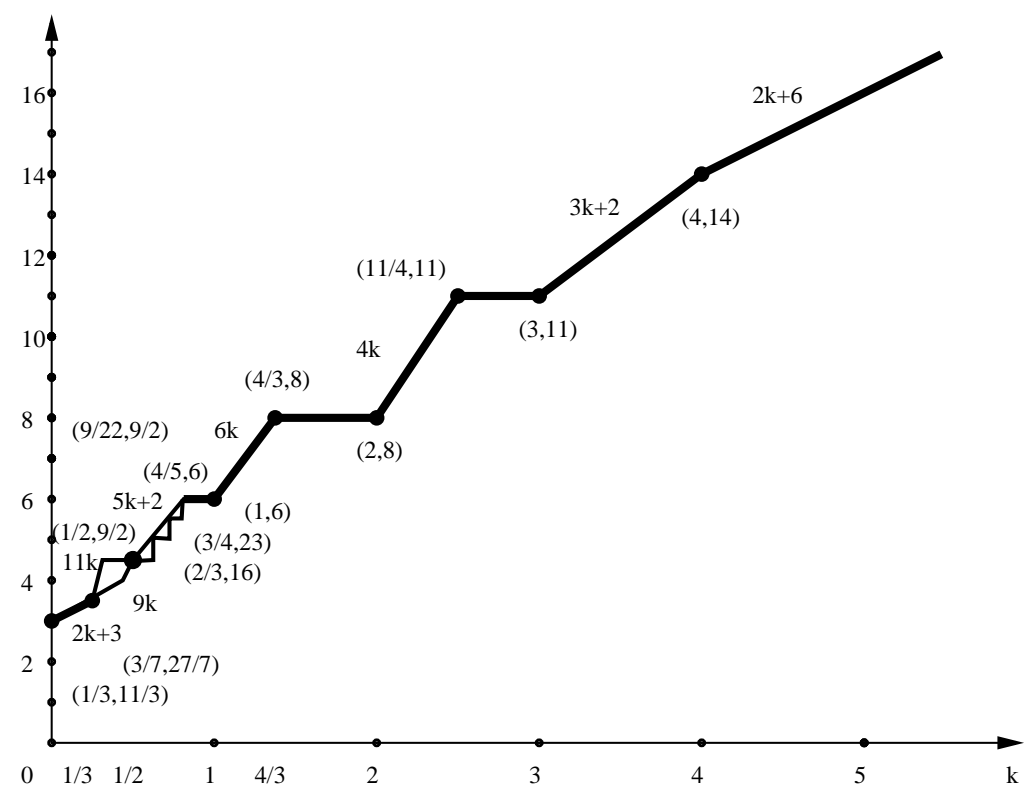

Figure 1: $\lambda\left(\Gamma_{\triangle} ; k, 1\right)$.

labeling problem for the triangular lattice for real numbers $k \geq 1$, and we give our best current bounds for $k \leq 1$ (see Figure 3). In Section 6 we describe some cases of the proof of this result, to illustrate all of our methods. A full proof is too long to include here, so will be published separately.

Theorem 2. For real number $k \geq 0$, we have the following for the minimum span of any $L(k, 1)$-labeling of the triangular lattice $\Gamma_{\triangle}$ :

$$
\lambda\left(\Gamma_{\triangle} ; k, 1\right) \begin{cases}=3 & \text { if } k=0 \\ =2 k+3 & \text { if } 0 k \leq \frac{1}{3} \\ \in[2 k+3,11 k] & \text { if } \frac{1}{3} \leq k \leq \frac{9}{22} \\ \in[9 k, 11 k] & \text { if } \frac{9}{22} \leq k \leq \frac{11}{27} \\ \in\left[9 k, \frac{9}{2}\right] & \text { if } \frac{11}{27} \leq k \leq \frac{1}{2} \\ \in\left[\frac{9}{2}, 5 k+2\right] & \text { if } \frac{1}{2} \leq k \leq \frac{2}{3} \\ \in\left[\frac{16}{3}, 5 k+2\right] & \text { if } \frac{2}{3} \leq k \leq \frac{3}{4} \\ \in\left[\frac{23}{4}, 5 k+2\right] & \text { if } \frac{3}{4} \leq k \leq \frac{4}{5} \\ =6 & \text { if } \frac{4}{5} \leq k \leq 1 \\ =6 k & \text { if } 1 \leq k \leq \frac{4}{3} \\ =8 & \text { if } \frac{4}{3} \leq k \leq 2 \\ =4 k & \text { if } 2 \leq k \leq \frac{11}{4} \\ =11 & \text { if } \frac{11}{4} \leq k \leq 3 \\ =3 k+2 & \text { if } 3 \leq k \leq 4 \\ =2 k+6 & \text { if } k \geq 4\end{cases}
$$

\section{The Square Lattice}

Inside cities, due to high buildings which are obstacles in the signal path (as well as a limited range

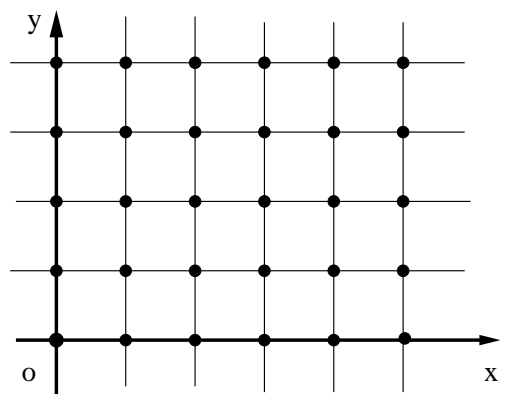

Figure 4: The Square Lattice $\Gamma_{\square}$

of a cell), a Manhattan fashion cellular system [4] can be modeled by the square lattice $\Gamma_{\square}$ (see Figure 4). Many graphs corresponding to cellular systems are the induced subgraphs of the square lattice or the triangular lattice.

Theorem 3 presents our full solution of the problem of determining $\lambda\left(\Gamma_{\square} ; k, 1\right)$ for real numbers $k \geq 0$. (We have no space to include the proof here.) Previously, Calamoneri [7] independently gave the minimum (integer) span $\lambda\left(\Gamma_{\square} ; k_{1}, k_{2}\right)$ for integers $k_{1} \geq 3 k_{2}$, as well as bounds when $k_{2} \leq k_{1} \leq 3 k_{2}$. It must be noted that the earlier paper [6] (an extended abstract) gave results for planar lattices that are not always correct. One such error example is that Theorem 2 in $[6]$ implies $\lambda\left(\Gamma_{\square} ; 3,2\right) \geq 12$. To the contrary, a labeling of van den Heuvel, Leese, 


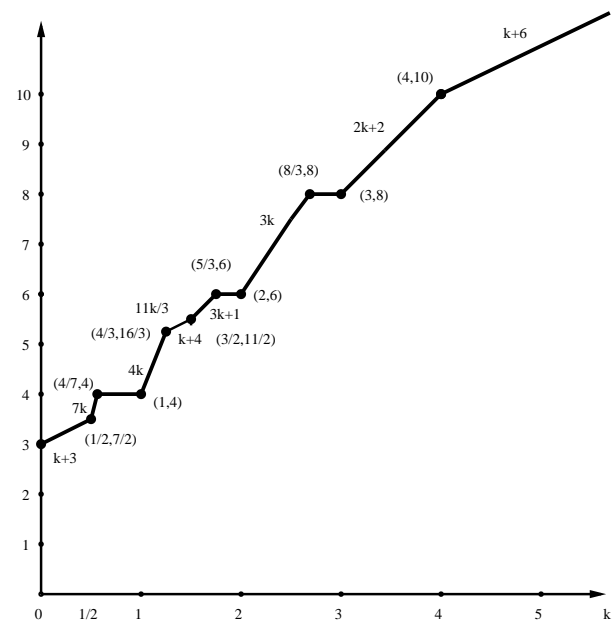

Figure 5: $\lambda\left(\Gamma_{\square} ; k, 1\right)$

and Shepherd [14] shows that $\lambda\left(\Gamma_{\square} ; 3,2\right) \leq 11$ : for any vertex with integer coordinates $(i, j)$, one assigns the label $(3 i+5 j) \quad(\bmod 12)$ in $\{0, \ldots, 11\}$.

Theorem 3. For real $k \geq 0$ we have the following minimum span:

$$
\lambda\left(\Gamma_{\square} ; k, 1\right)= \begin{cases}k+3 & \text { if } 0 \leq k \leq \frac{1}{2} \\ 7 k & \text { if } \frac{1}{2}<k \leq \frac{4}{7} \\ 4 & \text { if } \frac{4}{7} \leq k<1 \\ 4 k & \text { if } \leq k \leq \frac{4}{3} \\ k+4 & \text { if } \frac{4}{3}<k \leq \frac{3}{2} \\ 3 k+1 & \text { if } \frac{3}{2}<k \leq \frac{5}{3} \\ 6 & \text { if } \frac{5}{3} \leq k \leq 2 \\ 3 k & \text { if } 2<k \leq \frac{8}{3} \\ 8 & \text { if } \frac{8}{3} \leq k \leq 3 \\ 2 k+2 & \text { if } 3 \leq k \leq 4 \\ k+6 & \text { if } k \geq 4\end{cases}
$$

\section{The Hexagonal Lattice}

One may place the transmitters at nodes in the hexagonal lattice $\Gamma_{H}$ (see Figure 6), which is the dual of the triangular lattice. Calamoneri [7] gives the minimum span for the hexagonal lattice for integers $k_{1} \geq 2 k_{2}$ and the bounds for $k_{2} \leq k_{1} \leq 2 k_{2}$. We finish all the cases for real numbers $k \geq 0$ in [16].

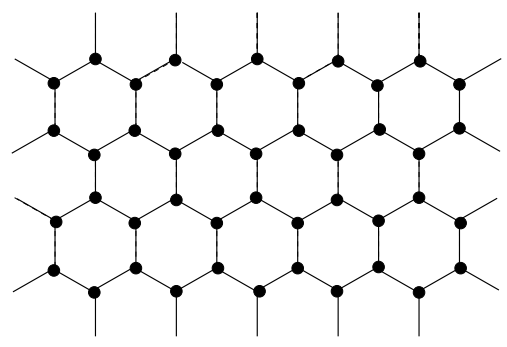

Figure 6: The Hexagonal Lattice $\Gamma_{H}$

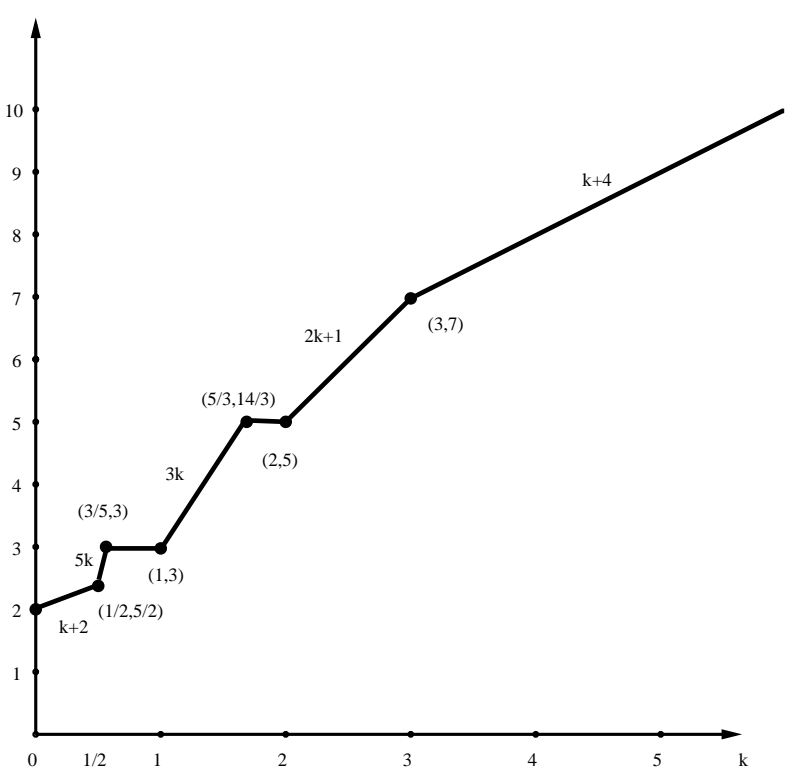

Figure $7: \lambda\left(\Gamma_{H} ; k, 1\right)$

Theorem 4. For real $k \geq 0$ we have the following minimum span:

$$
\lambda\left(\Gamma_{H} ; k, 1\right) \begin{cases}=k+2 & \text { if } 0 \leq k \leq \frac{1}{2} \\ =5 k & \text { if } \frac{1}{2} \leq k \leq \frac{3}{5} \\ =3 & \text { if } \frac{3}{5} \leq k \leq 1 \\ =3 k & \text { if } 1 \leq k \leq \frac{5}{3} \\ =5 & \text { if } \frac{5}{3} \leq k \leq 2 \\ =2 k+1 & \text { if } 2 \leq k \leq 3 \\ =k+4 & \text { if } k \geq 3\end{cases}
$$

\section{Proofs for the Triangular Lattice}

Generally, we get upper bounds by constructing feasible labelings and lower bounds by deriving contradictions on induced subgraphs for labelings of smaller span. Lemma 7 below is also useful in obtaining bounds. Here we present proofs of bounds in Theorem 2 for various cases. 


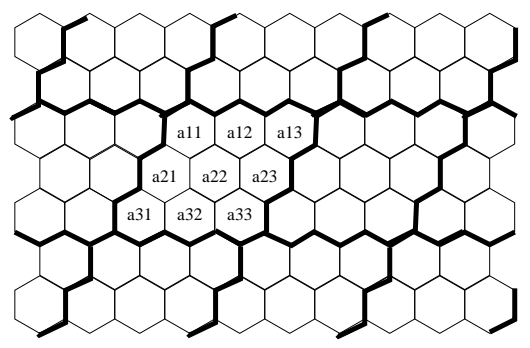

Figure 8: The Matrix Labeling

One construction method is to tile the whole lattice by a labeled parallelogram described by a matrix of labels.

Definition. Given an $m \times n$ labeling ma$\operatorname{trix} A:=\left[a_{i, j}\right]$, we label point $(i, j)$ by $a_{n-j(\bmod n), i+1(\bmod m)}$, for $i, j \in \mathbb{Z}$.

For example, if we have labeling matrix $A$ :

$$
A=\left[\begin{array}{lll}
a_{11} & a_{12} & a_{13} \\
a_{21} & a_{22} & a_{23} \\
a_{31} & a_{32} & a_{33}
\end{array}\right]
$$

Then Figure 8 shows how the labels are assigned, where $a_{3,1}$ is at the vertex with coordinates $(0,0)$ in the triangular lattice. The whole lattice is tiled with copies of the $3 \times 3$ tile shown:

Proposition 5. For $3 \leq k \leq 4, k \in \mathbb{R}$, we have $\lambda\left(\Gamma_{\triangle} ; k, 1\right) \leq 3 k+2$.

Proof: We extend the labeling from [11, 19], used for $k=3,4$, to real numbers $k, 3 \leq k \leq 4$, as defined by the $3 \times 4$ labeling matrix

$$
A=\left[\begin{array}{cccc}
3 k & 0 & k & 2 k \\
1 & k+1 & 2 k+1 & 3 k+1 \\
k+2 & 2 k+2 & 3 k+2 & 2
\end{array}\right]
$$

Proposition 6. For $k \geq 4, k \in \mathbb{R}$, we have $\lambda\left(\Gamma_{\triangle} ; k, 1\right) \leq 2 k+6$.

Proof: We extend the integer labelings from [5, $8,11,19,21$ ], used for $k \geq 4$, to all reals $k \geq 4$, as defined by the labeling matrix

$$
A=\left[\begin{array}{ccc}
2 k+5 & 0 & k+4 \\
1 & k+2 & 2 k+6 \\
k+3 & 2 k+4 & 2
\end{array}\right]
$$

Note if we find some upper (or lower) bound for some $k_{1}=a$, then we can get some upper (or lower) bounds for $k_{1} \leq a$ or $k_{1} \geq a$. For the case $p=2$, we
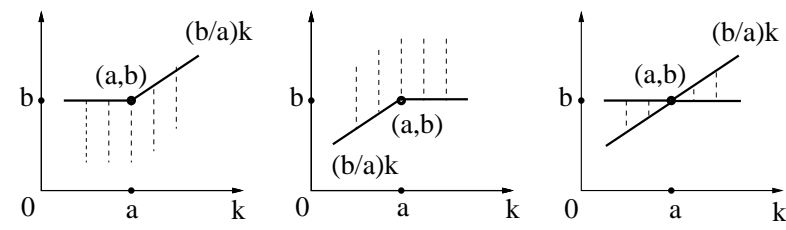

Figure $9: \lambda(G ; k, 1)$ have the following results (see Figure 9), by which we prove the bounds later .

Lemma 7. Let $a>0$.

If $\lambda(G ; a, 1) \leq b$, then

$$
\lambda(G ; k, 1) \leq \begin{cases}b & \text { if } 0 \leq k \leq a \\ \frac{b}{a} k & \text { if } k \geq a\end{cases}
$$

If $\lambda(G ; a, 1) \geq b$, then

$$
\lambda(G ; k, 1) \geq \begin{cases}\frac{b}{a} k & \text { if } 0 \leq k \leq a \\ b & \text { if } k \geq a\end{cases}
$$

In particular, if $\lambda(G ; a, 1)=b$, then $\frac{b}{a} k \leq \lambda(G ; k, 1) \leq b$ for $k \leq a$, while $b \leq \lambda(G ; k, 1) \leq \frac{b}{a} k$ if $k \geq a$.

Proof: If $\lambda(G ; a, 1) \leq b$, we have:

For $0 \leq k \leq a$, the result follows from the fact $\lambda(G ; k, 1)$ is nondecreasing.

For $k \geq a$, we have $\lambda(G ; k, 1) \leq \lambda\left(G ; k, \frac{k}{a}\right)=$ $\frac{k}{a} \lambda(G ; a, 1) \leq \frac{b}{a} k$.

The proof is similar, if $\lambda(G ; a, 1) \geq b$.

For positive integers $k_{1}, k_{2}$, if we have a feasible labeling $f \in L\left(k_{1}, k_{2}\right)$ with arithmetic progression $f(i, j) \equiv a i+b j \quad(\bmod l)$ for some positive integers $a, b, l$, then $\lambda\left(\Gamma_{\triangle} ; k_{1}, k_{2}\right) \leq l-1$. Hence we get $\lambda\left(\Gamma_{\triangle} ; k, 1\right) \leq(l-1) / k_{2}$, where $k=k_{1} / k_{2}$. We wrote a computer program to find feasible $L\left(k_{1}, k_{2}\right)$ labelings by arithmetic progressions, as we shall see below, which are then extended by Lemma 7 .

We need some notation. Given a vertex $v$, let $B_{7}\left(B_{17}, B_{37}\right.$ resp.) be the induced subgraph of $\Gamma_{\triangle}$ on all vertices which are at distance at most one (two, three resp.) from the vertex $v$.

Proposition 8 [15]. We have $\lambda\left(\Gamma_{\triangle} ; \frac{1}{2}, 1\right) \leq \frac{9}{2}$. Hence, $\lambda(G ; k, 1) \leq \frac{9}{2}$ if $0 \leq k \leq 1 / 2$.

Proof : We get the upper bound $\lambda\left(\Gamma_{\triangle} ; 1,2\right) \leq 9$ by labeling point $(i, j)$ by $i+4 j \quad(\bmod 10)$, taking the representative in $\{0, \ldots, 9\}$.

Proposition 9. We have $\lambda\left(\Gamma_{\triangle} ; 1,1\right)=6$. Hence, $\lambda(G ; k, 1) \leq \begin{cases}6 & \text { if } \frac{4}{5} \leq k \leq 1 \\ 6 k & \text { if } 1 \leq k \leq \frac{4}{3}\end{cases}$

Proof: The lower bound on $\lambda\left(\Gamma_{\triangle} ; 1,1\right)$ comes by checking that $\lambda\left(B_{7} ; 1,1\right)=6$. The upper bound 
is achieved by defining the labeling $f(i, j) \equiv i+$ $3 j(\bmod 7)$, where we choose the representative in $\{0, \ldots, 6\}$.

Proposition 10. We have $\lambda\left(\Gamma_{\triangle} ; 2,1\right) \leq 8$. Hence, $\lambda(G ; k, 1) \leq \begin{cases}8 & \text { if } \frac{4}{3} \leq k \leq 2 \\ 4 k & \text { if } 2 \leq k \leq \frac{11}{4}\end{cases}$

Proof: We get the upper bound by defining the integer labeling $f(i, j) \equiv 2 i+5 j(\bmod 9)$, which is unique by the symmetry of the Triangular Lattice.

We have $\lambda\left(\Gamma_{\triangle} ; 3,1\right) \leq 11$ by Proposition 5 , which implies the following by Lemma 7 :

Proposition 11. We have $\lambda\left(\Gamma_{\triangle} ; k, 1\right) \leq 11$, if $\frac{11}{4} \leq k \leq 3$.

We verify the lower bounds by contradiction and Lemma 7. We demonstrate two main methods of proof. The first method, for integers $k_{1}, k_{2}$, involves the successive elimination of possible labels, until a contradiction is reached. Sometimes we wrote a computer program to check all possible labelings of a fixed subgraph using a specified label set. We also used symmetry to reduce the number of cases we need to check:

The Symmetry Argument [5]. Let $G$ be a graph and $k_{1}, k_{2}, \ldots, k_{p} \in[0, \infty)$. If there exists a $L\left(k_{1}, k_{2}, \ldots, k_{p}\right)(G)$ labeling $f$ using label $x$, then there exists a $L\left(k_{1}, k_{2}, \ldots, k_{p}\right)(G)$ labeling using label $\operatorname{span}(f)-x$ with the same span, where $\operatorname{span}(f)=\max \{f(v)\}-\min \{f(v)\}$, for all vertices $v \in V(G)$.

We drew some ideas from [24] for the proof of the following important case.

Proposition 12 [16]. We have $\lambda\left(\Gamma_{\triangle} ; 4,3\right) \geq 24$, so that $\lambda\left(\Gamma_{\triangle} ; \frac{4}{3}, 1\right)=8$. Hence, $\lambda\left(\Gamma_{\triangle} ; k, 1\right)= \begin{cases}6 k & \text { if } \frac{3}{4} \leq k \leq \frac{4}{3} \\ 8 & \text { if } \frac{4}{3} \leq k \leq 2\end{cases}$

Proof: It suffices to prove $\lambda\left(\Gamma_{\triangle} ; 4,3\right) \geq 24$. Assume $\lambda\left(\Gamma_{\triangle} ; 4,3\right)<24$. By the $D$-set Theorem, there exists an optimal labeling $f \in L(4,3)\left(\Gamma_{\triangle}\right)$ with the smallest label 0 and all labels integers between 0 and 23.

Claim 1. Labeling $f$ cannot use label 3.

Proof of Claim 1: Assume $f$ uses label 3 at some vertex $v$. By the conditions, the six distinct labels around $v$ belong to $\{7,8, \ldots, 23\}$, the difference between any pair of them is at least 3 .

Consider all possible labelings on $B_{7}$ with center label 3. By computer program, we find only five feasible labelings of $B_{17}$, and none of these could be extended to $B_{37}$, and no such $f$ exists.
By the Symmetry Argument, labeling $f$ cannot use label $20=23-3$, which is the span of $f$ less 3 . Now $f$ has no labels 3 or 20 , and we continue (these claims must be proven sequentially).

Claim 2. Labeling $f$ cannot use label 7 .

Proof of Claim 2: Assume $f$ uses label 7 at some vertex $v$. Let the six distinct labels used around $v$ be $x_{1}<x_{2} \cdots<x_{6}$. Since $f \in$ $L(4,3)\left(\Gamma_{\triangle}\right)$, we have that $x_{i+1} \geq x_{i}+3$ for $i=$ $1,2, \ldots, 5$. If $x_{1}>7$, then $x_{1} \geq 11$, which gives $x_{6} \geq 26$, contradicting the span of $f$ being 23 . Hence, $x_{1}<7$, which forces $x_{1} \leq 2$, as 3 is excluded, while $x_{2} \geq 11, x_{3} \geq 14, x_{4} \geq 17, x_{5} \geq 21$ (because no label is 20 ), and $x_{6} \geq 24$, a contradiction.

By symmetry, $f$ has no label $3,7,16,20$. We continue to successively eliminate potential labels for $f$ until we are stuck. The proofs of the next three claims are similar:

Claim 3. Labeling $f$ cannot use label 6 nor $17=23-6$.

Claim 4. Labeling $f$ cannot use label 10 nor $13=23-10$.

Claim 5. Labeling $f$ cannot use label 11 nor $12=23-11$.

Now the set of all possible labels is: $\{0,1,2,4,5,8,9,14,15,18,19,21,22,23\}$.

We cannot find seven distinct labels for subgraph $B_{7}$, such that the difference between any two of them is at least 3 . It contradicts the existence of $f$. Thus $\lambda\left(\Gamma_{\triangle} ; 4,3\right) \geq 24$.

One of the winning teams in the Modeling contest, Goodwin, Johnston and Marcus (2000) [11], proved the following values for integer labelings, though their proof did not appear in the published version, due to space limitations. They presented labelings that achieved these values. Here we present a sketch of their lower bound arguments.

Proposition 13 [11]. For integers $k \geq 4$, we have $\lambda\left(\Gamma_{\triangle} ; k, 1\right)=2 k+6$. Moreover, for integers $k_{1}>6 k_{2}>0$, we have $\lambda\left(\Gamma_{\triangle} ; k_{1}, k_{2}\right)=2 k_{1}+6 k_{2}$.

Sketch of proof of lower bound: Similar to the proof of Proposition 12, we can show that $\lambda\left(\Gamma_{\triangle} ; k, 1\right) \geq 2 k+6$ for integer $k \geq 4$, i.e., $\lambda\left(\Gamma_{\triangle} ; k_{1}, k_{2}\right) \geq 2 k_{1}+6 k_{2}$ for integers $k_{1} \geq 4 k_{2}$ such that $k_{2}$ divides $k_{1}$.

Consider any integers $k_{1}>6 k_{2}>0$. Let $0<$ $m \leq k_{2}$ be such that $k_{1}+m \equiv 0 \quad\left(\bmod k_{2}\right)$. By the previous result, $\lambda\left(\Gamma_{\triangle} ; k_{1}+m, k_{2}\right) \geq 2 k_{1}+2 m+6 k_{2}$.

Assume for contradiction that $\lambda\left(\Gamma_{\triangle} ; k_{1}, k_{2}\right)<$ $2 k_{1}+6 k_{2}$. Let $f \in L\left(k_{1}, k_{2}\right)(G)$ be an optimal labeling. Define labeling $f_{1}$ by $f_{1}(v)=f(v)+m\left\lfloor\frac{f(v)}{k_{1}}\right\rfloor$. 
We can check that $f_{1} \in L\left(k_{1}+m, k_{2}\right)(G)$ with $\operatorname{span}\left(f_{1}\right)<\operatorname{span}(f)+m\left\lfloor\operatorname{span}(f) / k_{1}\right\rfloor<\left(2 k_{1}+6 k_{2}\right)+$ $m\left\lfloor\left(2 k_{1}+6 k_{2}\right) / k_{1}\right\rfloor=2 k_{1}+2 m+6 k_{2}$, which contradicts $\operatorname{span}\left(f_{1}\right) \geq \lambda\left(\Gamma_{\triangle} ; k_{1}+m, k_{2}\right) \geq 2 k_{1}+2 m+6 k_{2}$.

Similar lower bound arguments are used by Jin and Yeh [15] in another case, $L(1,2)$. Combining this with Proposition 8, and extending it by Lemma 7, gives us:

Proposition 14. We have $\lambda\left(\Gamma_{\triangle} ; \frac{1}{2}, 1\right) \geq \frac{9}{2}$. Hence, $\lambda\left(\Gamma_{\triangle} ; k, 1\right)= \begin{cases}9 k & \text { if } \frac{3}{7} \leq k \leq \frac{1}{2} \\ \frac{9}{2} & \text { if } \frac{1}{2} \leq k \leq \frac{3}{4}\end{cases}$

The second lower bound proof method involves the successive removal of intervals of possible labels until there is a contradiction. Calamoneri [6] already gave the same corresponding result for integer labelings, $\lambda\left(\Gamma_{\triangle} ; k_{1}, k_{2}\right)$ for integers $k_{1}, k_{2}$ with $3 k_{2} \leq k_{1} \leq 4 k_{2}$. Her lower bound method involves looking at a small induced subgraph of the lattice and checking cases according to the numerical order of the labels. This is similar to the method devised independently by Georges and Mauro for labeling trees [10].

Proposition 15. For $3 \leq k<4$, we have $\lambda\left(\Gamma_{\triangle} ; k, 1\right) \geq 3 k+2$. Hence $\lambda\left(\Gamma_{\triangle} ; k, 1\right)=3 k+2$.

Proof: Assume $\lambda\left(\Gamma_{\triangle} ; k, 1\right)=l<3 k+2$, By the $D$-set Theorem, there is an optimal labeling $f \in$ $L(k, 1)\left(\Gamma_{\triangle}\right)$ with $\operatorname{span}(f)=l<3 k+2$ and the smallest label zero.

Claim 1. Labeling $f$ cannot use labels in the interval $[k-1, k)$.

Proof of Claim 1: Assume $f(v) \in[k-1, k)$ for some $v \in V\left(\Gamma_{\triangle}\right)$. The six distinct labels around $v$ are $\geq f(v)+k$. Since the induced subgraph by $N(v)$ is cycle $C_{6}$ on six vertices, $\lambda\left(\Gamma_{\triangle} ; k, 1\right) \geq f(v)+$ $k+\lambda\left(C_{6} ; k, 1\right) \geq(k-1)+k+(k+3)=3 k+2$ (because $\lambda\left(C_{6} ; k, 1\right)=k+3$ for $k \geq 3$ ). It gives a contradiction. $\square$

Thus, $f(v) \notin[k-1, k)$ for all $v \in V\left(\Gamma_{\triangle}\right)$. By the symmetry argument, $f(v) \notin(l-k, l-k+1]$ for all $v \in V\left(\Gamma_{\triangle}\right)$.

Now, $f(v) \in I_{1} \cup I_{2} \cup I_{3}=[0, k-1) \cup[k, l-$ $k] \cup(l-k+1, l]$ for all $v \in V\left(\Gamma_{\triangle}\right)$, where $I_{1}=$ $[0, k-1), I_{2}=[k, l-k], I_{3}=(l-k+1, l]$. Then $\left|I_{1}\right|=k-1<k,\left|I_{3}\right|=k-1<k$.

By similar arguments we can successively prove these two claims:

Claim 2. Labeling $f$ cannot use labels in the interval $[k, k+1)$.

Claim 3. Labeling $f$ cannot use labels in the interval $[k+1, k+2)$.
Thus, $f(v) \notin[k-1, k+2)$ for all $v \in V\left(\Gamma_{\triangle}\right)$. By the symmetry argument, $f(v) \notin(l-k-2, l-k+1]$ for all $v \in V\left(\Gamma_{\triangle}\right)$. Now, $f(v) \in I_{1} \cup I_{2}^{\prime} \cup I_{3}=$ $[0, k-1) \cup[k+2, l-k-2] \cup(l-k+1, l]$ for all vertices $v$, where $I_{2}^{\prime}=[k+2, l-k-2]$. Then $\left|I_{2}^{\prime}\right| \leq$ $l-2 k-4<k-2<2$ for $k<4$.

Let $u$ be a vertex with label zero. Among the six labels around $u$ (no two closer than 1 apart), no label is in $I_{1}=[0, k-1)$ (because $\left.\left|I_{1}\right|<k\right)$, at most two labels are in $I_{2}^{\prime}=[k+2, l-k-2]$ (because $\left|I_{2}^{\prime}\right|<$ $2)$, and at most three labels are in $I_{3}=(l-k+1, l]$ (because $\left|I_{3}\right|<k$, these labels cannot be adjacent to each other). But this only accounts for five of the six neighbors of $v$, a contradiction.

\section{Further Research}

In general, once the minimum spans of feasible labelings have been determined, we would like to describe all optimal labelings. For the triangular lattice, we have found all integer optimal labelings for the cases $L(0,1), L(1,1)$, and $L(2,1)$, and we are investigating many other cases. We want to find properties of optimal labelings of the lattices that help us to find optimal labelings in straightforward ways. We will discuss these properties and the relations among them in a later paper.

Bertossi, Pinotti, Tan [4] gave a nontrivial result with conditions at distance $3, \lambda\left(\Gamma_{\triangle} ; 2,1,1\right)=11$. It would useful for researchers to obtain other real number values $\lambda\left(G ; k_{1}, k_{2}, k_{3}\right)$ when $G$ is a regular lattice.

Since the graph labeling problem comes from the radio channel assignment problem in wireless communications, we wish to promote its applications by communication with the field engineers. So we offer our results here.

\section{References}

[1] S. Anand, A. Sridharan, K. N. Sivarajan, Performance Analysis of Channelized Cellular Systems with Dynamic Channel Allocation, IEEE Transactions on Vehicular Technology, 52 (4), 847-859, 2003.

[2] Piera Barcaccia, Maurizio A. Bonuccelli, Polynomial Time Optimal Algorithms for Time Slot Assignment of Variable Bandwidth Systems, IEEE/ACM Transactions on Networking, 2 (3), 247-251, June, 1994.

[3] A. A. Bertossi, M. A. Bonuccelli, Code Assignment for Hidden Terminal Interference 
Avoidance in Multihop Packet Radio Networks, IEEE/ACM Trans. on Networking, 3, 441-449, 1995.

[4] A. A. Bertossi, Cristina M. Pinotti, Richard B. Tan, Channel Assignment with Separation for Inference Avoidance in Wireless Networks, IEEE Transactions on Parallel and Distributed Systems, 14 (3), 222-235, 2003.

[5] Robert E. Broadhurst, William J. Shanahan, Michael D. Steffen, "We're Sorry, You're Outside the Coverage Area", The UMAP Journal, 21 (3), 327-342, Fall, 2000.

[6] Tiziana Calamoneri, Exact Solution of a Class of Frequency Assignment Problems in Cellular Networks and other Regular Girds, (Extended Abstract) 8th Italian Conference on Theoretical Computer Science (ICTCS2003), Lect. Notes in Comp. Science, 2841, 150-162, 2003

[7] Tiziana Calamoneri, Exact Solution of a Class of Frequency Assignment Problems in Cellar Networks and Other Regular Girds, Preprint, 2004.

[8] Daniel J. Durand, Jacob M. Kline, Kevin M. Woods, Groovin' with the Big Band(width), The UMAP Journal, 21 (3), 357-368, Fall, 2000 .

[9] A. Gamst, Homogeneous Distribution of Frequencies in a Regular Hexagonal Cell System, IEEE Transactions on Vehicular Technology VT-31, 132-144, 1982.

[10] John P. Georges, David W. Mauro, Labeling Trees with a Condition at Distance Two, Discrete Mathematics, 269, 127-148, July, 2003.

[11] Justin Goodwin, Dan Johnston, Adam Marcus, Radio Channel Assignments, The UMAP Journal, 21 (3), 379-386, Fall, 2000.

[12] Jerrold R. Griggs, Roger K. Yeh, Labelling Graphs with a Condition at Distance 2, SIAM J. Disc. Math., 5 (4), 586-595, Nov., 1992.

[13] Jerrold R. Griggs, Author/Judge's Commentary; The Outstanding Channel Assignment Papers, The UMAP Journal, 21 (3), 379-386, Fall, 2000.
[14] J. van den Heuvel, R. A. Leese, M. A. Shepherd, Graph Labeling and Radio Channel Assignment, J. Graph Theory, 29, 263-283, 1998.

[15] Xiaohua Teresa Jin, Roger K. Yeh, Graph Distance-Dependent Labeling Related to Code Assignment in Computer Networks, Naval Research Logistics, in press.

[16] Xiaohua Teresa Jin, Ph.D. Dissertation. Mathematics Department, University of South Carolina, in preparation.

[17] A. Kramling, M. Scheibenbogen, B. Walke, Dynamic Channel Allocation in Wireless ATM Networks, Wireless Networks, 2, 381398, 2002.

[18] Robert A. Leese, A Unified Approach to the Assignment of Radio Channels on a Regular Hexagonal Grid, IEEE Transactions on Vehicular Technology, 46 (4), 968-979, Nov., 1997.

[19] Jeffrey Mintz, Aaron Newcomer, James C. Price, Channel Assignment Model: The Span Without a Face, The UMAP Journal, 21 (3), 311-326, Fall, 2000.

[20] S. Ramanathan, A Unified Framework and Algorithm for Channel Assignment in Wireless Networks, Wireless Networks, 5, 81-94, 1999.

[21] Chu Rui, Xiu Baoxin, Zhong Ruidi, Utilize the Limited Frequency Resources Efficiently, The UMAP Journal, 21 (3), 343-356, Fall, 2000.

[22] Saswati Sarkar, Kumar N. Sivarajan, Channel Assignment Algorithms Satisfying Cochannel and Adjacent Channel Reuse, IEEE Transactions on Vehicular Technology, 51 (5), 954967, Sept., 2002.

[23] Roger M. Whitaker, Steve Hurley, Stuart M. Allen, Optimising Channel Assignments for Private Mobile Radio Networks in the UHF 2 Band, Wireless Networks, 8, 587-595, 2002.

[24] Daoyun Zhu, Aiju Shi, Optimal Channel Assignments, Preprint, Aug. 28, 2001. 